\title{
CYP3A5*3 and POR*28 Genetic Variants Influence the Required Dose of Tacrolimus in Heart Transplant Recipients
}

\author{
Dorothea Lesche, MSc, * Vilborg Sigurdardottir, MD, PhD, $\neq$ Raschid Setoud, PhD, $\neq$ \\ Markus Oberhänsli, MD, $\neq$ Thierry Carrel, MD, $\S$ Georg M. Fiedler, MD, * Carlo R. Largiadèr, PhD,* \\ Paul Mohacsi, Prof, $\neq$ and Johanna Sistonen, PhD*
}

\begin{abstract}
Background: After heart transplantation (HTx), the interindividual pharmacokinetic variability of immunosuppressive drugs represents a major therapeutic challenge due to the narrow therapeutic window between over-immunosuppression causing toxicity and underimmunosuppression leading to graft rejection. Although genetic polymorphisms have been shown to influence pharmacokinetics of immunosuppressants, data in the context of HTx are scarce. We thus assessed the role of genetic variation in CYP3A4, CYP3A5, POR, NR1I2, and $A B C B 1$ acting jointly in immunosuppressive drug pathways in tacrolimus (TAC) and ciclosporin (CSA) dose requirement in HTx recipients.
\end{abstract}

Methods: Associations between 7 functional genetic variants and blood dose-adjusted trough $\left(\mathrm{C}_{0}\right)$ concentrations of TAC and CSA at $1,3,6$, and 12 months after HTx were evaluated in cohorts of 52 and 45 patients, respectively.

Results: Compared with CYP3A5 nonexpressors (*3/*3 genotype), CYP3A5 expressors $(* 1 / * 3$ or $* 1 / * 1$ genotype) required around 2.2 to 2.6-fold higher daily TAC doses to reach the targeted $\mathrm{C}_{0}$ concentration at all studied time points $(P \leq 0.003)$. Additionally, the $P O R^{*} 28$ variant carriers showed higher dose-adjusted TAC- $\mathrm{C}_{0}$ concentrations at all time points resulting in significant differences at 3 $(P=0.025)$ and 6 months $(P=0.047)$ after HTx. No significant associations were observed between the genetic variants and the CSA dose requirement.

Conclusions: The $C Y P 3 A 5^{*} 3$ variant has a major influence on the required TAC dose in HTx recipients, whereas the $P O R^{*} 28$ may additionally contribute to the observed variability. These results support the importance of genetic markers in TAC dose optimization after HTx.

Received for publication January 29, 2014; accepted April 3, 2014.

From the *Institute of Clinical Chemistry, University Hospital (Inselspital Bern), and University of Bern; †Traduate School for Cellular and Biomedical Sciences, University of Bern; and Departments of $\ddagger$ Cardiology, and $\S$ Cardiovascular Surgery, Swiss Cardiovascular Centre, University Hospital (Inselspital Bern), Bern, Switzerland.

Supported by a grant from the Swiss National Science Foundation (31003A_138285 to C.R.L.) and by the K. Huber-Steiner Foundation.

The authors declare no conflict of interest.

D. Lesche and V. Sigurdardottir are co-primary authors.

Supplemental digital content is available for this article. Direct URL citations appear in the printed text and are provided in the HTML and PDF versions of this article on the journal's Web site (www.drug-monitoring.com).

Correspondence: Paul Mohacsi, Professor of Heart Failure, Department of Cardiology, Swiss Cardiovascular Centre, University Hospital (Inselspital Bern), CH-3010 Bern, Switzerland (e-mail: paul.mohacsi@insel.ch).

Copyright (C) 2014 by Lippincott Williams \& Wilkins
Key Words: heart transplantation, tacrolimus, ciclosporin, pharmacogenomics

(Ther Drug Monit 2014;36:710-715)

\section{INTRODUCTION}

Immunosuppressive drug therapy after organ transplantation is complicated by the narrow therapeutic window of the drugs combined with high and unpredictable interindividual variability in response to standard administered doses. It has been increasingly recognized that, along with factors like kidney function and drug interactions, genetic variation contributes to this large variability in response. ${ }^{1-3}$ Although genetic factors influencing immunosuppressive response have been more widely studied in kidney and liver transplant patients, data in the context of heart transplantation (HTx) are scarce. Because management of HTx recipients has unique characteristics, such as higher level of required immunosuppression combined with risk of serious toxic complications or graft failure, studies focusing specifically on this patient population are clinically important. Furthermore, the effect of a patient's genetic variation on drug pharmacokinetics may differ between heart and other solid organ transplants, such as the kidneys and the liver, where donor genetic variation could also affect drug metabolism and elimination.

Calcineurin inhibitors (CNIs) are currently still the basis of immunosuppressive protocols after HTx. ${ }^{4}$ Drug-metabolizing enzymes cytochrome P450 (CYP) 3A4 and CYP3A5 and the efflux transporter P-glycoprotein [ATP-binding cassette B1 (ABCB1)], which are responsible for the first-pass metabolism and systemic clearance of CNIs, are the major determinants of their pharmacokinetics. ${ }^{5}$ The majority of the previous studies have focused on the common CYP $3 A 5^{*} 3$ (rs776746) variant, which results in a cryptic splice site and nonfunctional CYP3A5 protein. CYP3A5 expressors (ie, carriers of $* 1 * 1$ or $* 1 * 3$ genotype) have been shown to require higher daily dose of both tacrolimus (TAC) and ciclosporin (CSA) to reach the targeted blood trough $\left(\mathrm{C}_{0}\right)$ concentrations compared with CYP3A5 nonexpressors $(* 3 / * 3) \cdot{ }^{6-8}$ Additionally, polymorphisms in the $A B C B 1$ gene have been widely studied but with controversial results. ${ }^{7,9-11}$

Recently, a novel functional polymorphism in CYP3A4 (CYP3A4*22; rs35599367) was described and associated with lower CNI dose requirement in transplant patients. ${ }^{12-15}$ Furthermore, polymorphisms in the P450 oxidoreductase (POR 
gene) and pregnane $\mathrm{X}$ receptor (PXR, NR1I2 gene) have been shown to modulate the activity of CYP3A enzymes and to alter the pharmacokinetics of TAC in kidney transplant patients. ${ }^{16,17}$ Studying multiple polymorphisms in genes acting together on an immunosuppressive drug pathway instead of single variants may provide new insight into factors influencing the response. ${ }^{18}$

In this study, our main aim was to evaluate the effect of functional polymorphisms in 5 candidate genes on TAC and CSA dose requirement during the first year after transplantation. Because altered drug pharmacokinetics may influence the treatment efficacy and toxicity, we additionally explored the potential role of genetic variation in the treatment outcome in terms of allograft rejection and renal function.

\section{MATERIALS AND METHODS}

\section{Study Population}

This study was a retrospective observational cohort study including HTx recipients from the University Hospital Bern (Inselspital), who underwent transplantation between the years 1994 and 2012 and were alive in April 2012. All consecutive patients were invited to participate in the study during their regular follow-up visits, except patients fulfilling the exclusion criteria (ie, patients having received more than 1 transplant or being younger than 16 years). All transplant recipients received perioperative immunosuppressive therapy with $5 \mathrm{mg} / \mathrm{kg}$ of azathioprine (AZA) and $1000 \mathrm{mg}$ of methylprednisolone. Antithymocyte globulin (4-5 $\mathrm{mg} / \mathrm{kg}$ ) was initiated within 12 hours posttransplant and continued for up to a maximum of 5 days tailored by CD3 cell count. All patients received a CNI for early postoperative immunosuppression and for maintenance treatment in combination with prednisone $\left(0.8 \mathrm{mg} \cdot \mathrm{kg}^{-1} \cdot \mathrm{d}^{-1}\right.$ tapered to 0.15 $\mathrm{mg} \cdot \mathrm{kg}^{-1} \cdot \mathrm{d}^{-1}$ within 5 months) and AZA or mycophenolate mofetil (replaced AZA in 2007). In case of severe early postoperative kidney dysfunction, CNI was tapered or replaced by a mammalian target of rapamycin (mTOR) inhibitor. The targeted $\mathrm{C}_{0}$ concentration was $170-220 \mathrm{ng} / \mathrm{mL}$ (or $50-75$ $\mathrm{ng} / \mathrm{mL}$ in case of the combination with an mTOR inhibitor) for CSA and $8-14 \mathrm{ng} / \mathrm{mL}$ (or 3-5 $\mathrm{ng} / \mathrm{mL}$ in case of the combination with an mTOR inhibitor) for TAC. The targeted $\mathrm{C}_{0}$ concentrations for the mTOR inhibitors everolimus and sirolimus were 6-8 and 10-12 ng/mL, respectively. Protocol endomyocardial biopsy specimens were performed weekly during the first postoperative month. Thereafter, the intervals were extended depending on the individual clinical course. The endomyocardial biopsy results were classified according to the guidelines of the International Society of Heart and Lung Transplantation. ${ }^{19}$ Renal function was assessed by an estimate of the glomerular filtration rate (eGFR) calculated by the Chronic Kidney Disease Epidemiology Collaboration equation. $^{20}$ EDTA blood samples for genetic analyses were collected at the time of study recruitment. Clinical data, including patient characteristics, dosing and blood $\mathrm{C}_{0}$ concentrations of TAC and CSA, concomitant drug therapy (such as statins, calcium channel blockers, antibiotics, and antimycotics), and number of clinically relevant (grade $\geq 2 \mathrm{R}$ ) biopsy-proven allograft rejections at 1, 3, 6, and 12 months post-HTx, were retrieved from the patient charts. Patients' immunosuppressive therapy regimen at 1 month after HTx was used to assign patients into TAC and CSA treatment groups. All patients received an oral and a written description of the study protocol and signed a written informed consent. The study was approved by the Cantonal Ethics Committee of Bern (KEK 031/12).

\section{Dose-Adjusted Drug $\mathrm{C}_{0}$ Concentrations}

Blood concentration measurements of TAC and CSA were obtained from the routine therapeutic drug monitoring. The $\mathrm{C}_{0}$ concentrations were measured in whole blood samples using chemiluminescence microparticle immunoassays on the Architect i2000SRTM (Abbott Laboratories, Abbott Park, IL) since 2008. A microparticle immunoassay on the Abbott IMX was previously used for TAC and a chemiluminescence microparticle immunoassay for CSA on the same platform. Dose-adjusted drug $\mathrm{C}_{0}$ concentration was used as an outcome variable, as commonly done in other studies, ${ }^{7,8,11,13,21,22}$ and defined as drug $\mathrm{C}_{0}$ concentration per daily weight-adjusted drug dose $\left[(\mathrm{ng} / \mathrm{mL}) /\left(\mathrm{mg} \cdot \mathrm{kg}^{-1} \cdot \mathrm{d}^{-1}\right)\right]$.

\section{Selection of Genetic Variants and Genotyping}

Genetic variants were selected based on previous literature. Seven candidate polymorphisms in 5 genes shown to influence the metabolism or transport of TAC and/or CSA were selected. These included variants in drug-metabolizing enzymes: $C Y P 3 A 5^{*} 3$ (rs776746) and $C Y P 3 A 4^{*} 22$ (rs35599367) ${ }^{13}$; in proteins modifying the CYP enzyme activity: POR*28 (rs1057868) $)^{17}$ and NR1I2 $-25385 \mathrm{C}>\mathrm{T}$ (rs3814055) ${ }^{16}$; and in the efflux transporter P-glycoprotein: $A B C B 1 \quad$ c. $1236 \mathrm{C}>\mathrm{T} \quad(\mathrm{rs} 1128503), \quad$ c. $2677 \mathrm{G}>\mathrm{T} / \mathrm{A}$ $(\mathrm{rs} 2032582)$, and c.3435C $>\mathrm{T}(\operatorname{rs} 1045642){ }^{7,9,11}$

Genomic DNA was extracted from EDTA blood samples using QIAamp DNA Blood Midi Kit (Qiagen AG, Basel, Switzerland). Genetic variants were genotyped using TaqMan SNP and Drug Metabolism Genotyping Assays (Applied Biosystems, Foster City, CA): C_59013445_10 for CYP3A4*22, C_26201809_30 for CYP3A5*3, C_8890131_30 for $P O R^{*} 28$, C_27504984_30 for NR1I2 $-25385 \mathrm{C}>\mathrm{T}, \quad$ C_7586662_10 for $A B C B \overline{1} \quad$ c. $1236 \mathrm{C}>\mathrm{T}$, C_11711720C_30 and C_11711720D_40 for c. $2677 \mathrm{G}>\mathrm{T} /$ A, and C_7586657_20 for c.3435C $>$ T. Allelic discrimination was performed on a 7500 Fast Real-Time PCR System (Applied Biosystems) according to the manufacturer's protocol. Genotyping results of 21 patient samples representing different genotypes of the studied polymorphisms were confirmed by Sanger sequencing.

\section{Statistical Analyses}

Statistical analyses were performed using the Predictive Analytics Software statistics for Windows version 21.0 (SPSS, Inc, Chicago, IL) unless otherwise stated. Normal distribution of data was assessed by Kolmogorov-Smirnov test and data were presented as mean ( \pm SD) or as median (interquartile range) where appropriate. The dose-adjusted TAC- $\mathrm{C}_{0}$ and $\mathrm{CSA}-\mathrm{C}_{0}$ concentrations were normalized by logarithmic transformation. Genotype frequencies of the 
polymorphisms were tested for deviations from Hardy-Weinberg equilibrium using exact tests implemented in the software Arlequin v.3.5.1.3. ${ }^{23}$ Pairwise linkage disequilibrium between $A B C B 1$ polymorphisms was assessed by calculating the $D^{\prime}$ coefficient $^{24}$ and tested as implemented in the package "genetics" in the R software (http://www.r-project.org). $A B C B 1$ haplotypes were inferred for the 3 loci (showing $\left.D^{\prime} \geq 0.89 ; P<0.001\right)$ using the Excoffier-Laval-Balding algorithm implemented in Arlequin. ${ }^{25}$ The associations of genetic variants with dose-adjusted TAC- $\mathrm{C}_{0}$ and $\mathrm{CSA}-\mathrm{C}_{0}$ concentrations were evaluated at $1,3,6$, and 12 months after HTx using linear regression analysis adjusted for clinical factors, namely, age, sex, eGFR, daily prednisone dose, and calcium channel blocker use. The information on the use of other drugs inhibiting (ie, fluconazole, voriconazole) or inducing (ie, rifampin) CYP3A enzymes was omitted from the analysis because only single patients received these drugs at the studied time points. An additive model was assumed for all genetic variants. Percentage change in the untransformed dose-adjusted $\mathrm{C}_{0}$ concentration was determined by back-transforming estimated regression coefficients using equation $\left[\left(e^{\beta}-1\right) \times 100\right]$. The Mann-Whitney $U$ test was used for pairwise comparisons of continuous variables and the Fisher exact test for comparisons of categorical variables. Repeatedmeasures analysis of variance was used to compare the eGFR values between genotype groups during the first year post-HTx. Two-sided nominal $P$ values $<0.05$ were considered statistically significant. No correction for multiple testing was performed because of the exploratory nature of this study.

\section{RESULTS}

The study population consisted of 104 HTx recipients (78 males) with a mean age of 47.4 years, the majority being whites $(95 \%)$ (Table 1). The mean posttransplant follow-up time was $7.6( \pm 4.9)$ years. At 1 month after HTx, immunosuppressive therapy regimen was based on TAC for 52 patients and on CSA for 45 patients. Seven patients on a CNI-free therapy regimen (Table 1) were excluded from further data analyses. The genotype frequencies of the studied polymorphisms (Table 2) were in accordance with Hardy-Weinberg equilibrium $(P>0.05)$, and the observed allele frequencies were similar to the previously described frequencies in whites. ${ }^{26}$

\section{Associations of Genetic Variants With Dose-Adjusted Drug- $C_{0}$ Concentrations}

The $C Y P 3 A 5^{*} 3$ variant was strongly associated with the dose-adjusted TAC- $\mathrm{C}_{0}$ concentration during the first year after HTx (Table 3). ${ }^{27}$ CYP3A5 expressors had lower doseadjusted TAC- $\mathrm{C}_{0}$ concentrations at all studied time points $(P \leq 0.002$; Fig. 1; Table 4$)$ and required 2.2- to 2.6-fold higher drug dose to reach the targeted concentration compared with CYP3A5 nonexpressors (Table 4). Additionally, the $P O R^{*} 28$ variant carriers showed higher dose-adjusted TAC- $\mathrm{C}_{0}$ concentrations at all time points resulting in significant differences at $3(P=0.025)$ and 6 months $(P=0.047)$ after HTx (Table 3). In contrast to TAC, no consistent associations between the genetic variants and the dose-adjusted $\mathrm{CSA}-\mathrm{C}_{0}$ concentration during the first year of therapy were
TABLE 1. Baseline Characteristics of the Study Population $(\mathrm{n}=104)$

\begin{tabular}{lc}
\hline Variable & \\
\hline Male sex, $\mathrm{n}(\%)$ & $78(75)$ \\
Age at the time of transplant $(\mathrm{yrs})$ & $70.4( \pm 14.3)$ \\
Weight at the time of transplant $(\mathrm{kg})$ & \\
Ethnicity, $\mathrm{n}(\%)$ & $99(95)$ \\
White & $3(3)$ \\
Asian & $2(2)$ \\
Other & \\
Primary cause of transplantation, $\mathrm{n}(\%)$ & $46(44)$ \\
Dilated cardiomyopathy & $39(38)$ \\
Coronary artery disease & $19(18)$ \\
Other & $73.5( \pm 31.2)$ \\
eGFR before transplantation $\left(\mathrm{mL} / \mathrm{min} / 1.73 \mathrm{~m}^{2}\right)$ & \\
Drug therapy at $1 \mathrm{mo}^{2}$ after transplantation, $\mathrm{n}(\%)$ & $80(77)$ \\
Statins* & $0.37(0.32-0.46)$ \\
Prednisone $\left(\mathrm{mg} \cdot \mathrm{kg}^{-1} \cdot \mathrm{d}^{-1}\right)$ & $43(41)$ \\
CSA + MMF or AZA & $2(2)$ \\
CSA + SRL & $1(1)$ \\
TAC & $46(44)$ \\
TAC + MMF or AZA & $1(1)$ \\
TAC + SRL & $4(4)$ \\
TAC + ERL + MMF & $7(7)$ \\
ERL + MMF &
\end{tabular}

Continuous data are presented as mean $( \pm \mathrm{SD})$ or as median (interquartile range) where appropriate.

*The use of statins gradually increased: at 12 months after transplantation 93 (89\%) patients were on statin therapy.

ERL, everolimus; MMF, mycophenolate mofetil; SRL, sirolimus.

observed (see Table, Supplemental Digital Content 1, http://links.lww.com/TDM/A89).

\section{Relationship Between CYP3A5*3 Genotype and TAC Treatment Outcome}

As a strong effect of the $C Y P 3 A 5^{*} 3$ variant on the required TAC dose to reach the targeted $\mathrm{C}_{0}$ concentration was observed, its impact on the treatment outcome in terms of allograft rejection and renal function during the first year after HTx was evaluated. Although no significant differences in the incidence of clinically relevant biopsy-proven allograft rejection in relation to the $C Y P 3 A 5^{*} 3$ genotype were observed, the number of patients experiencing early allograft rejection (ie, within the first 3 months after HTx) was higher among CYP3A5 expressors 46\% (6/13) compared with nonexpressors $28 \%(11 / 39)(P=0.31)$. The eGFR decreased in the TAC treatment cohort from $75.9( \pm 35.3) \mathrm{mL} / \mathrm{min} / 1.73 \mathrm{~m}^{2}$ at 1 month to $64.9( \pm 27.8) \mathrm{mL} / \mathrm{min} / 1.73 \mathrm{~m}^{2}$ at 12 months after HTx. CYP $3 A 5^{*} 3$ genotype had no influence on the development of the eGFR values $(P=0.68)$.

\section{DISCUSSION}

Although substantial variability exists in the immunosuppressive response of heart transplant patients, predictive markers to optimize the therapy in the early postoperative 
TABLE 2. Genotype Frequencies

\begin{tabular}{|c|c|c|}
\hline Genotype & $\begin{array}{c}\text { TAC Treatment Group } \\
(\mathrm{n}=52), \mathrm{n}(\%)\end{array}$ & $\begin{array}{c}\text { CSA Treatment Group } \\
(\mathrm{n}=45), \mathrm{n}(\%)\end{array}$ \\
\hline \multicolumn{3}{|l|}{ CYP $3 A 4 * 22$} \\
\hline$* 1 / * 1$ & $51(98)$ & $39(87)$ \\
\hline$* 1 * 22$ & $1(2)$ & $6(13)$ \\
\hline$* 22 / * 22$ & $0(0)$ & $0(0)$ \\
\hline \multicolumn{3}{|l|}{ СYР $3 A 5 * 3$} \\
\hline$* 1 / * 1$ & $1(2)$ & $0(0)$ \\
\hline$* 1 / * 3$ & $12(23)$ & $9(20)$ \\
\hline$* 3 / * 3$ & $39(75)$ & $36(80)$ \\
\hline \multicolumn{3}{|l|}{$P O R^{*} 28$} \\
\hline$* 1 / * 1$ & $27(52)$ & $22(49)$ \\
\hline$* 1 / 28$ & $18(35)$ & $19(42)$ \\
\hline$* 28 / * 28$ & $7(13)$ & $4(9)$ \\
\hline \multicolumn{3}{|c|}{ NRII2 $-25385 \mathrm{C}>\mathrm{T}$} \\
\hline $\mathrm{C} / \mathrm{C}$ & $22(42)$ & $17(38)$ \\
\hline $\mathrm{C} / \mathrm{T}$ & $26(50)$ & $19(42)$ \\
\hline $\mathrm{T} / \mathrm{T}$ & $4(8)$ & $9(20)$ \\
\hline \multicolumn{3}{|c|}{$A B C B 1$ c $1236 \mathrm{C}>\mathrm{T}$} \\
\hline $\mathrm{C} / \mathrm{C}$ & $19(37)$ & $14(31)$ \\
\hline $\mathrm{C} / \mathrm{T}$ & $21(40)$ & $22(49)$ \\
\hline $\mathrm{T} / \mathrm{T}$ & $12(23)$ & $9(20)$ \\
\hline \multicolumn{3}{|c|}{$A B C B 1$ c. $2677 \mathrm{G}>\mathrm{T} / \mathrm{A}$} \\
\hline $\mathrm{G} / \mathrm{G}$ & $15(29)$ & $11(24)$ \\
\hline $\mathrm{G} / \mathrm{A}$ & $3(6)$ & $3(7)$ \\
\hline $\mathrm{G} / \mathrm{T}$ & $21(40)$ & $23(51)$ \\
\hline $\mathrm{T} / \mathrm{A}$ & $1(2)$ & $0(0)$ \\
\hline $\mathrm{T} / \mathrm{T}$ & $12(23)$ & $8(18)$ \\
\hline \multicolumn{3}{|c|}{$A B C B 1$ c. $3435 \mathrm{C}>\mathrm{T}$} \\
\hline $\mathrm{C} / \mathrm{C}$ & $13(25)$ & $10(22)$ \\
\hline $\mathrm{C} / \mathrm{T}$ & $22(42)$ & $20(45)$ \\
\hline $\mathrm{T} / \mathrm{T}$ & $17(33)$ & $15(33)$ \\
\hline
\end{tabular}

*l indicates wild type (see http://www.cypalleles.ki.se/).

phase are currently lacking. In this study, we assessed the role of 7 genetic variants in 5 candidate genes acting jointly in the metabolism and transport pathways of immunosuppressants in the dose requirement of TAC and CSA in a cohort of 104
HTx recipients. Our main results suggest that the $C Y P 3 A 5^{*} 3$ variant has a major influence on the required TAC dose in HTx recipients, whereas the $P O R^{*} 28$ may additionally contribute to the observed variability.

Our results showed that the CYP3A5 expressors had significantly lower dose-adjusted $\mathrm{TAC}^{-\mathrm{C}_{0}}$ concentrations compared with CYP3A5 nonexpressors at all studied time points requiring around 2.2- to 2.6-fold higher daily $\mathrm{TAC}$ doses to reach the targeted blood $\mathrm{C}_{0}$ concentration. A similar effect of the $C Y P 3 A 5^{*} 3$ variant on TAC dose requirement has been shown also in the previous studies on HTx recipients, based on cohorts of 15 and 65 adult patients ${ }^{21,28}$ and 37 and 54 pediatric patients. ${ }^{11,22}$ This study, however, was the first to investigate the influence of the $P O R^{*} 28$ variant on TAC and CSA dose requirement in HTx recipients.

POR is a modulator of CYP enzyme activity. It is a microsomal flavoprotein that transfers electrons from NADPH to CYP enzymes, enabling their catalytic activity. ${ }^{29}$ Of known polymorphisms in the POR gene, the $P O R^{*} 28$ variant, which induces an amino acid substitution A503V, has been the most widely investigated. ${ }^{30}$ Although in vitro studies have shown that this variant leads to decreased catalytic activity, the effect in vivo seems to vary depending on the enzyme and substrate analyzed. ${ }^{31}$ De Jonge et $a^{17}$ reported in their study on 298 de novo kidney transplant recipients a significantly higher TAC dose requirement in carriers of the $P O R^{*} 28$ during the first year of therapy but only if they were CYP3A5 expressors $(\mathrm{n}=52)$. Similarly, a lower TAC exposure in carriers of the $P O R^{*} 28$ was described exclusively in CYP3A5 expressors in a recent study on 43 pediatric kidney transplant recipients ${ }^{32}$ and in a pharmacokinetic study on 71 healthy Chinese male volunteers receiving a single oral TAC dose of $2 \mathrm{mg} .{ }^{33}$ Our results showing significantly higher dose-adjusted $\mathrm{TAC}^{-\mathrm{C}_{0}}$ concentrations in carriers of the $P O R^{*} 28$, which indicates a lower TAC dose requirement in these patients, are thus in line with the previous in vitro data but in contrast to the in vivo studies on TAC exposure. Subanalysis of the $P O R^{*} 28$ in different $C Y P 3 A 5^{*} 3$ genotype groups was not meaningful in our TAC study cohort including only 13 CYP3A5 expressors of whom 4 carried the $P O R^{*} 28$ variant. Larger HTx patient cohorts

TABLE 3. Associations of Genetic Variants and Normalized TAC Dose-Adjusted $\mathrm{C}_{0}$ Concentrations

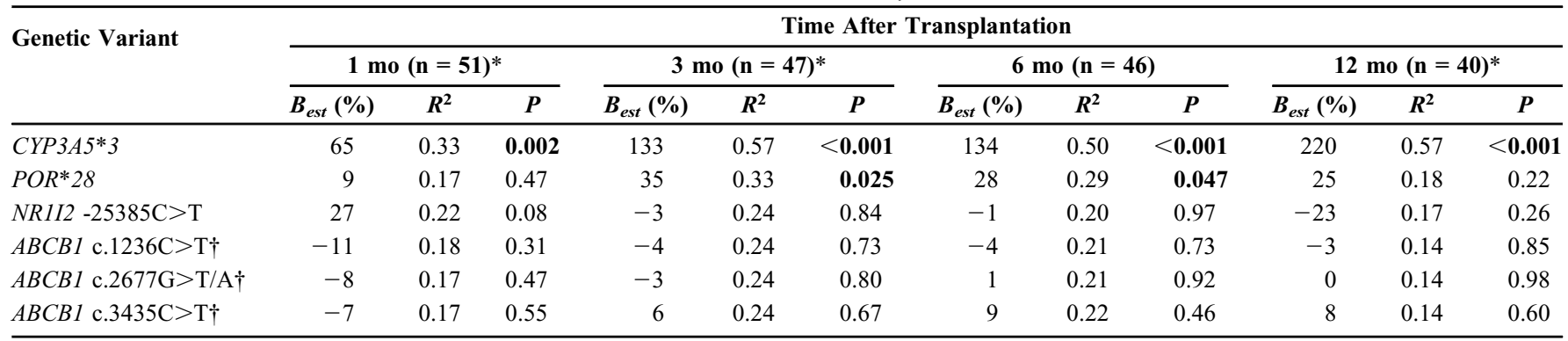

Linear regression analysis of each genetic variant was performed using an additive genetic model and adjusted for age, sex, eGFR, prednisone dose, and calcium channel blocker use. Bold font indicates $P<0.05$.

*TAC dose-adjusted $\mathrm{C}_{0}$ concentration was missing for 1 patient at 1,3 , and 12 months after HTx.

$\dagger$ Rare $A B C B 1$ c.2677A allele was grouped with the wild-type G allele in the analysis based on its predicted functional effect. ${ }^{27} A B C B 1$ polymorphisms were also analyzed on a haplotype level but no significant associations were observed.

$B_{\text {est }}$, estimated percentage change in the outcome variable; $R^{2}$, coefficient of determination of full model. 


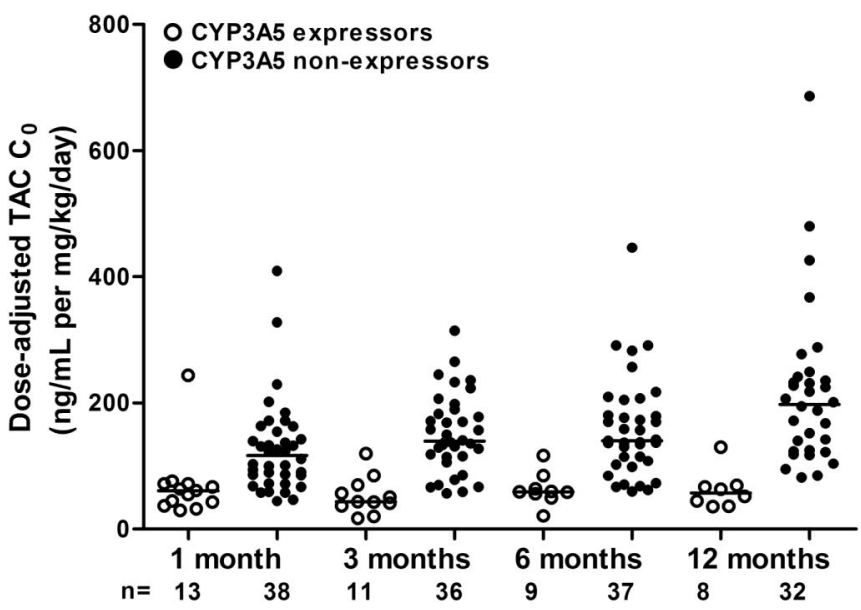

FIGURE 1. Dose-adjusted TAC- $\mathrm{C}_{0}$ concentrations in blood during 1 year after transplantation in CYP3A5 expressors $(* 1 / * 1$ or $* 1 / * 3$ genotype) and nonexpressors $(* 3 / * 3)$. The horizontal line represents the median value. The difference between CYP3A5 expressors and nonexpressors was significant at each time point $(P \leq 0.001)$.

are needed to further elucidate the effect of this polymorphism, in combination with $C Y P$ genetic variants, on TAC pharmacokinetics.

In contrast to TAC, no consistent associations between the genetic variants and the CSA dose requirement were observed. Previous studies on the effect of the $C Y P 3 A 5^{*} 3$ variant on CSA dose requirement have yielded controversial results. A meta-analysis by $\mathrm{Zhu}$ et $\mathrm{al}^{8}$ based on 1742 kidney transplant patients showed a significant association between CYP3A5 nonexpressors and higher dose-adjusted CSA- $\mathrm{C}_{0}$ concentration, indicative of lower dose requirement in these patients compared with CYP3A5 expressors. However, no effect or an opposite effect has also been reported. ${ }^{34-36}$ Based on a statistical power analysis, this study including 45 patients on a CSA-based therapy could detect a $35 \%$ change in the doseadjusted $\mathrm{CSA}-\mathrm{C}_{0}$ concentration between CYP3A5 expressors and nonexpressors with a power of $80 \%$ at an alpha level of 0.05 . Therefore, although our results do not rule out an effect of $C Y P 3 A 5^{*} 3$ genotype on CSA dose requirement, it is not a strong predictor as in the case of TAC.

Because of the relatively small patient cohort, this study was statistically underpowered to detect even large differences in clinical outcomes between CYP3A5 expressors and nonexpressors. However, it is worth noting that a higher number of CYP3A5 expressors (46\%) experienced early allograft rejection in our TAC patient cohort compared with CYP3A5 nonexpressors $(28 \%)(P=0.31)$. This is in agreement with Tang et $\mathrm{al}^{6}$ who reported in their meta-analysis including 1779 kidney and liver transplant patients on TAC-based therapy significantly higher rate of acute graft rejections in CYP3A5 expressors compared with nonexpressors over the first month after transplantation (odds ratio, 3.27 ; $95 \%$ CI, $1.57-6.81 ; P=0.002)$. The influence of higher daily TAC dose requirement and potentially longer doseadjustment time in CYP3A5 expressors on the risk of allograft rejection during the first months after HTx needs further evaluation.

The limitations of our study include a small cohort of patients who underwent transplantation during a period of 18 years. Changes in surgical techniques and clinical protocols within this time may have influenced the treatment outcomes in these patients. Clinical data, such as drug $\mathrm{C}_{0}$ concentrations, were retrospectively retrieved from the patient charts introducing potential errors to the data. However, we expect that these limitations are independent of a patient's genotype and thus should rather lead to an underestimation of the associations between the pharmacokinetics of immunosuppressants

TABLE 4. TAC Dose and $C_{0}$ Concentration in CYP3A5 Expressors and Nonexpressors

\begin{tabular}{|c|c|c|c|c|c|}
\hline & CYP3A5 Expressors & n* & CYP3A5 Nonexpressors & n* & $P$ \\
\hline \multicolumn{6}{|c|}{ Dose $\left(\mathrm{mg} \cdot \mathrm{kg}^{-1} \cdot \mathrm{d}^{-1}\right)$} \\
\hline $1 \mathrm{mo}$ & $0.24(0.16-0.35)$ & 11 & $0.11(0.09-0.17)$ & 35 & 0.003 \\
\hline $3 \mathrm{mo}$ & $0.22(0.17-0.28)$ & 8 & $0.09(0.06-0.13)$ & 32 & $<\mathbf{0 . 0 0 1}$ \\
\hline $12 \mathrm{mo}$ & $0.16(0.11-0.18)$ & 6 & $0.07(0.05-0.09)$ & 29 & $<\mathbf{0 . 0 0 1}$ \\
\hline \multicolumn{6}{|c|}{$\mathrm{C}_{0}(\mathrm{ng} / \mathrm{mL})$} \\
\hline $6 \mathrm{mo}$ & $13.9(11.8-14.9)$ & 6 & $11.8(10.2-13.5)$ & 34 & 0.12 \\
\hline $12 \mathrm{mo}$ & $9.5(7.9-12.8)$ & 6 & $12.6(11.2-15.5)$ & 29 & 0.07 \\
\hline \multicolumn{6}{|c|}{$\begin{array}{l}\text { Dose-adjusted } \mathrm{C}_{0}[(\mathrm{ng} / \mathrm{mL}) / \\
\left.\quad\left(\mathrm{mg} \cdot \mathrm{kg}^{-1} \cdot \mathrm{d}^{-1}\right)\right]\end{array}$} \\
\hline $1 \mathrm{mo}$ & $61.2(43.0-71.9)$ & 11 & $122.2(85.2-154.0)$ & 35 & 0.001 \\
\hline $3 \mathrm{mo}$ & $43.1(37.9-77.7)$ & 8 & $139.1(116.1-186.8)$ & 32 & $<\mathbf{0 . 0 0 1}$ \\
\hline
\end{tabular}

Data are presented as median (interquartile range). Bold font indicates $P<0.05$.

*Small number of patients on a combination therapy with an mTOR inhibitor were excluded from the analysis because of different target $\mathrm{C}_{0}$ concentration. 
and genetic factors. Finally, the number of studied genetic variants was limited, and thus, important genetic effects may have remained undetected in this study.

\section{CONCLUSIONS}

We have shown that the $C Y P 3 A 5^{*} 3$ variant has a major influence on the required TAC dose in HTx recipients. Because this finding is supported by other studies on HTx recipients, adjusting the starting dose of TAC based on the $C Y P 3 A 5^{*} 3$ genotype would require evaluation in a multicentre prospective study. The $P O R^{*} 28$ variant was additionally identified in our study as a new candidate marker influencing TAC pharmacokinetics in HTx recipients. Our results support the importance of genetic markers in TAC dose optimization after HTx.

\section{ACKNOWLEDGMENTS}

The authors would like to thank Tanja Fröhlich and Mojgan Yazdanpanah for their help with the data analyses.

\section{REFERENCES}

1. Thervet E, Anglicheau D, Legendre C, et al. Role of pharmacogenetics of immunosuppressive drugs in organ transplantation. Ther Drug Monit. 2008;30:143-150.

2. Elens L, Bouamar R, Shuker N, et al. Clinical implementation of pharmacogenetics in kidney transplantation: CNIs in the starting blocks. $\mathrm{Br}$ J Clin Pharmacol. 2014;77:715-728.

3. Hesselink DA, Bouamar R, Elens L, et al. The role of pharmacogenetics in the disposition of and response to tacrolimus in solid organ transplantation. Clin Pharmacokinet. 2014;53:123-139.

4. Stehlik J, Edwards LB, Kucheryavaya AY, et al. The Registry of the International Society for Heart and Lung Transplantation: 29th official adult heart transplant report 2012. J Heart Lung Transplant. 2012;31: 1052-1064.

5. Monchaud C, Marquet P. Pharmacokinetic optimization of immunosuppressive therapy in thoracic transplantation: part I. Clin Pharmacokinet. 2009; 48:419-462.

6. Tang HL, Xie HG, Yao Y, et al. Lower tacrolimus daily dose requirements and acute rejection rates in the CYP3A5 nonexpressers than expressers. Pharmacogenet Genomics. 2011;21:713-720.

7. Terrazzino S, Quaglia M, Stratta $\mathrm{P}$, et al. The effect of CYP3A5 $6986 \mathrm{~A}>\mathrm{G}$ and $A B C B 13435 \mathrm{C}>\mathrm{T}$ on tacrolimus dose-adjusted trough levels and acute rejection rates in renal transplant patients: a systematic review and meta-analysis. Pharmacogenet Genomics. 2012;22: 642-645.

8. Zhu HJ, Yuan SH, Fang Y, et al. The effect of CYP3A5 polymorphism on dose-adjusted cyclosporine concentration in renal transplant recipients: a meta-analysis. Pharmacogenomics $J$. 2011;11:237-246.

9. Fredericks S, Moreton M, Reboux S, et al. Multidrug resistance gene-1 (MDR-1) haplotypes have a minor influence on tacrolimus dose requirements. Transplantation. 2006;82:705-708.

10. Haufroid V, Mourad M, Van Kerckhove V, et al. The effect of CYP3A5 and $M D R 1$ (ABCB1) polymorphisms on cyclosporine and tacrolimus dose requirements and trough blood levels in stable renal transplant patients. Pharmacogenetics. 2004;14:147-154

11. Zheng H, Webber S, Zeevi A, et al. Tacrolimus dosing in pediatric heart transplant patients is related to $C Y P 3 A 5$ and $M D R 1$ gene polymorphisms. Am J Transplant. 2003;3:477-483.

12. Elens L, Bouamar R, Hesselink DA, et al. A new functional CYP3A4 intron 6 polymorphism significantly affects tacrolimus pharmacokinetics in kidney transplant recipients. Clin Chem. 2011;57:1574-1583.

13. Elens L, van Schaik RH, Panin N, et al. Effect of a new functional CYP3A4 polymorphism on calcineurin inhibitors' dose requirements and trough blood levels in stable renal transplant patients. Pharmacogenomics. 2011;12:1383-1396.

14. Wang D, Guo Y, Wrighton SA, et al. Intronic polymorphism in CYP3A4 affects hepatic expression and response to statin drugs. Pharmacogenomics J. 2011;11:274-286.

15. Gijsen VM, van Schaik RH, Elens L, et al. CYP3A4*22 and $C Y P 3 A$ combined genotypes both correlate with tacrolimus disposition in pediatric heart transplant recipients. Pharmacogenomics. 2013;14:1027-1036.

16. Benkali K, Premaud A, Picard N, et al. Tacrolimus population pharmacokinetic-pharmacogenetic analysis and Bayesian estimation in renal transplant recipients. Clin Pharmacokinet. 2009;48:805-816.

17. de Jonge $\mathrm{H}$, Metalidis $\mathrm{C}$, Naesens $\mathrm{M}$, et al. The $\mathrm{P} 450$ oxidoreductase $* 28$ SNP is associated with low initial tacrolimus exposure and increased dose requirements in CYP3A5-expressing renal recipients. Pharmacogenomics. 2011;12:1281-1291.

18. Evans WE, Relling MV. Moving towards individualized medicine with pharmacogenomics. Nature. 2004:429:464-468.

19. Stewart S, Winters GL, Fishbein MC, et al. Revision of the 1990 working formulation for the standardization of nomenclature in the diagnosis of heart rejection. J Heart Lung Transplant. 2005;24:1710-1720.

20. Levey AS, Stevens LA, Schmid CH, et al. A new equation to estimate glomerular filtration rate. Ann Intern Med. 2009;150:604-612.

21. Diaz-Molina B, Tavira B, Lambert JL, et al. Effect of $C Y P 3 A 5, C Y$ $P 3 A 4$, and $A B C B 1$ genotypes as determinants of tacrolimus dose and clinical outcomes after heart transplantation. Transplant Proc. 2012;44: 2635-2638.

22. Gijsen V, Mital S, van Schaik RH, et al. Age and CYP3A5 genotype affect tacrolimus dosing requirements after transplant in pediatric heart recipients. J Heart Lung Transplant. 2011;30:1352-1359.

23. Excoffier L, Lischer HE. Arlequin suite ver 3.5: a new series of programs to perform population genetics analyses under Linux and Windows. Mol Ecol Resour. 2010;10:564-667.

24. Lewontin RC. The interaction of selection and linkage. I. General considerations; heterotic models. Genetics. 1964;49:49-67.

25. Excoffier L, Laval G, Balding D. Gametic phase estimation over large genomic regions using an adaptive window approach. Hum Genomics. 2003;1:7-19.

26. The 1000 Genomes Project. Available at: http://www.1000genomes.org/. Accessed August 27, 2013.

27. Sakurai A, Onishi Y, Hirano $\mathrm{H}$, et al. Quantitative structure-activity relationship analysis and molecular dynamics simulation to functionally validate nonsynonymous polymorphisms of human $\mathrm{ABC}$ transporter ABCB1 (P-glycoprotein/MDR1). Biochemistry. 2007;46:7678-7693.

28. Kniepeiss D, Renner W, Trummer O, et al. The role of CYP3A5 genotypes in dose requirements of tacrolimus and everolimus after heart transplantation. Clin Transplant. 2011;25:146-150.

29. Zanger UM, Schwab M. Cytochrome P450 enzymes in drug metabolism: regulation of gene expression, enzyme activities, and impact of genetic variation. Pharmacol Ther. 2013;138:103-141.

30. Huang N, Agrawal V, Giacomini KM, et al. Genetics of P450 oxidoreductase: sequence variation in 842 individuals of four ethnicities and activities of 15 missense mutations. Proc Natl Acad Sci U S A. 2008; 105:1733-1738.

31. Miller WL, Agrawal V, Sandee D, et al. Consequences of $P O R$ mutations and polymorphisms. Mol Cell Endocrinol. 2011;336:174-179.

32. Gijsen VM, van Schaik RH, Soldin OP, et al. P450 oxidoreductase *28 $\left(P^{*}{ }^{*} 28\right)$ and tacrolimus disposition in pediatric kidney transplant recipients - a pilot study. Ther Drug Monit. 2014;36:152-158.

33. Zhang JJ, Zhang H, Ding XL, et al. Effect of the P450 oxidoreductase *28 polymorphism on the pharmacokinetics of tacrolimus in Chinese healthy male volunteers. Eur J Clin Pharmacol. 2013;69:807-812.

34. Chu XM, Hao HP, Wang GJ, et al. Influence of CYP3A5 genetic polymorphism on cyclosporine A metabolism and elimination in Chinese renal transplant recipients. Acta Pharmacol Sin. 2006;27: $1504-1508$

35. Fanta S, Niemi M, Jonsson $\mathrm{S}$, et al. Pharmacogenetics of cyclosporine in children suggests an age-dependent influence of $A B C B 1$ polymorphisms. Pharmacogenet Genomics. 2008;18:77-90.

36. Hesselink DA, van Schaik RH, van der Heiden IP, et al. Genetic polymorphisms of the CYP $3 A 4, C Y P 3 A 5$, and MDR-1 genes and pharmacokinetics of the calcineurin inhibitors cyclosporine and tacrolimus. Clin Pharmacol Ther. 2003;74:245-254. 